\title{
On Springback Prediction With Special Reference To Constitutive Modeling
}

\author{
P.-A. Eggertsen ${ }^{\mathrm{a}}$ and K. Mattiasson ${ }^{\mathrm{a}, \mathrm{b}}$ \\ ${ }^{a}$ Div. of Material and Computational Mechanics, Dept. of Applied Mechanics, Chalmers University of \\ Technology, SE-412 96 Göteborg, Sweden \\ e-mail:eggepera@chalmers.se,kjellm@chalmers.se \\ ${ }^{b}$ Dept. 91430, PV22, Volvo Cars Safety Center, SE-405 31 Göteborg, Sweden \\ e-mail:kmattias@volvocars.com
}

\begin{abstract}
The springback phenomenon that occur in thin metal sheets after forming is mainly a stress driven problem, and the magnitude is roughly proportional to the ratio between the magnitude of the residual stresses after forming and Young's modulus. An accurate prediction of residual stresses puts, however, high demands on the material modeling. A phenomenological plasticity model is made up of several ingredients, such as a yield criterion, a plastic hardening curve, a hardening law, and a model for the degradation of elastic stiffness due to plastic straining.

The authors have recently, Ref. [1], showed the importance of a correct modeling of a cyclic stress-strain behavior via a phenomenological hardening law, in order to obtain an accurate stress prediction. The main purposes of the present study are to study the influence of two other constitutive ingredients: The yield criterion and the material behavior during unloading.

The material behavior during unloading is evaluated by loading/unloading/reloading tension tests, where the material is unloaded/reloaded at specific plastic strain levels. The slope of the unloading curve is measured and a relation between the "unloading modulus" and the plastic strains is established.

In the current study, results for four different materials are accounted for. The springback of a simple U-bend is calculated for all the materials in the rolling-, transverse- and diagonal directions. From the results of these simulations, some conclusions regarding constitutive modeling for springback simulations are drawn.
\end{abstract}

Keywords: Hardening law, Springback, Bauschinger effect, yield surface, Simulation

PACS: $62.20 . \mathrm{fq}$

\section{INTRODUCTION}

In case of uniaxial tension, the transition from elastic to plastic state occurs when the stress level reaches the yield point of the material. For multiaxial stress state the criterion for transition from elastic to plastic state becomes more complex. A relationship between stresses is needed to specify when plastic flow occurs. Such a relationship is known as a yield criterion. Numerous yield criteria, describing the initial, anisotropic behavior of sheet metals, have been proposed in the literature. For a review of some recent criteria see Mattiasson and Sigvant [4].

During unloading of the material, i.e. removal of the forming tools, the amount of springback depends to a great extent on the elastic stiffness of the material. In classic plasticity theory, the unloading of a material after plastic deformation is assumed to be linearly elastic with the stiffness equal to Young's modulus. However, several experimental investigations have revealed that this is an incorrect assumption [2].

The main purpose of the present study is to evaluate the influence of the modeling of the two above mentioned effects on the springback prediction of thin metal sheets.

\section{MATERIALS}

Four different materials, frequently used in the car manufacturing industry, were considered in this study: Two DP600 steels from two different suppliers and with different thicknesses, a mild DX56 steel and, finally, a 220IF steel, classified somewhere in between the two latter material classes. However, in this report, only two of these materials will be accounted for. The mechanical properties of these two materials are presented in Table 1 below. 
TABLE 1. Material data for the steel grades included in the current study.

\begin{tabular}{|c|c|c|c|c|c|c|c|c|c|}
\hline Material & $\begin{array}{l}\sigma_{0} \\
{[\mathbf{M P a}]}\end{array}$ & $\begin{array}{l}\sigma_{45} \\
{[\mathrm{MPa}]}\end{array}$ & $\begin{array}{l}\sigma_{90} \\
{[\mathrm{MPa}]}\end{array}$ & $\begin{array}{l}\sigma_{\mathbf{b}} \\
{[\mathbf{M P a}]}\end{array}$ & $\mathbf{r}_{\mathbf{0}}$ & $\mathbf{r}_{45}$ & $\mathbf{r}_{90}$ & $\mathbf{r}_{\mathbf{b}}$ & $\begin{array}{l}\text { Thickness } \\
\text { [mm] }\end{array}$ \\
\hline TKS-DP600HF & 363.70 & 364.00 & 385.60 & 374.70 & 0.46 & 0.88 & 1.00 & 0.85 & 1.46 \\
\hline TKS-220IF & 226.70 & 226.10 & 238.90 & 276.98 & 1.60 & 2.10 & 2.42 & 0.96 & 0.96 \\
\hline
\end{tabular}

\section{YIELD FUNCTIONS FOR ANISOTROPIC METAL SHEETS}

Numerous yield criteria, describing the initial, anisotropic behavior of sheet metals, have been proposed in the literature. For a review of some recent criteria see Mattiasson and Sigvant [4]. The appearance of the yield criterion is known to have a significant effect on the calculated strain and stress distribution, and is also very important for the prediction of strain localization and fracture. In this study, three different criteria of different complexity are considered.

\section{Hill's Quadratic Yield Criterion from 1948}

The Hill'48 yield criterion [7], is a generalisation of the well known von Mises yield criterion. Originally it was presented for a full stress space, but it can be reduced to plane stress and will then take the form:

$$
\sigma_{x x}^{2}-\frac{2 r_{0}}{1+r_{0}} \sigma_{x x} \sigma_{y y}+\frac{r_{0}\left(1+r_{90}\right)}{r_{90}\left(1+r_{0}\right)} \sigma_{y y}^{2}+\frac{r_{0}+r_{90}}{r_{90}\left(1+r_{0}\right)}\left(2 r_{45}+1\right) \sigma_{x y}^{2}=\bar{\sigma}^{2}
$$

where $r_{0}, r_{45}$ and $r_{90}$ are Lankford coefficients describing the anisotropy.

\section{Barlat-Lian's Yield Criterion from 1989 (Yld89)}

The Barlat-Lian (Yld89) yield criterion, [8], can be viewed as a generalization of Hosford's, [9], yield condition for plane stress to states of orthotropic anisotropy:

$$
f=a\left|k_{1}+k_{2}\right|^{M}+a\left|k_{1}-k_{2}\right|^{M}+c\left|2 k_{2}\right|^{M}=\bar{\sigma}^{M}
$$

where

$$
\begin{aligned}
& k_{1}=\frac{\sigma_{x x}+h \sigma_{y y}}{2} \\
& k_{2}=\sqrt{\left(\frac{\sigma_{x x}-h \sigma_{y y}}{2}\right)^{2}+p^{2} \sigma_{x y}^{2}}
\end{aligned}
$$

The parameters $a, c, h$ and $p$ are material constants that can be determined from experimental tension tests. The exponent $M$ is related to the crystallographic structure of the material, and it is set to 6 for steel grades and 8 for aluminum alloys according to the work by Logan and Hosford [9]. It can be noticed that for $M=2$ the Barlat-Lian yield criterion is reduced to the Hill' 48 criterion.

\section{The Banabic/Aretz/Barlat Eight Parameter Model}

The eight parameter yield criterion by Banabic et al. [10] (see also Barlat et al. [12], Aretz [13] and Mattiasson and Sigvant [4]) is an extension of the Barlat-Lian (Yld89) criterion such that

$$
\bar{\sigma}(L, K, N, P, Q, R, S, T, \boldsymbol{\sigma})=\left[\frac{1}{2}\left(|\Gamma+\Psi|^{M}+|\Gamma-\Psi|^{M}+|2 \Lambda|^{M}\right)\right]^{\frac{1}{M}}
$$

where the functions $\Gamma, \Psi$ and $\Lambda$ are defined as 


$$
\begin{aligned}
& \Gamma=\frac{L \sigma_{x x}+K \sigma_{y y}}{2} \\
& \Psi=\sqrt{\frac{\left(N \sigma_{x x}-P \sigma_{y y}\right)^{2}}{4}+Q^{2} \sigma_{x y} \sigma_{x y}} \\
& \Lambda=\sqrt{\frac{\left(R \sigma_{x x}-S \sigma_{y y}\right)^{2}}{4}+T^{2} \sigma_{x y} \sigma_{x y}}
\end{aligned}
$$

The coefficients $L, K, N, P, Q, R, S$ and $T$ are material parameters. All these parameters affect the shape of the yield surface. However, most influence on the yield surface shape has the exponent $M$, even though it is not directly determined from experimental results. Just as for the Barlat-Lian criterion $M$ is chosen to 6 or 8 for steel grades and aluminum alloys, respectively. The eight parameters are determined such that the model reproduces the experimental characteristics of the orthotropic sheet metal as good as possible. Those experimental characteristics are three uniaxial yield stresses $\left(Y_{0}, Y_{45}\right.$ and $\left.Y_{90}\right)$, the equi-biaxial yield stress $\left(Y_{\mathrm{b}}\right)$, the three anisotropic coefficients $\left(r_{0}, r_{45}\right.$ and $\left.r_{90}\right)$ and, finally, the equi-biaxial $\mathrm{r}$-value $\left(r_{\mathrm{b}}\right)$.

It can easily be seen from Equations 2-5 that the Banabic/Aretz criterion can be reduced to the Barlat-Lian criterion by choosing a suitable, reduced set of parameters. Consequently, it can also easily be reduced to the Hill 48 ' criterion.

\section{KINEMATIC HARDENING PARAMETERS DETERMINED FROM A THREE-POINT BENDING TEST}

The authors have recently, Ref. [1], showed the importance of a correct modeling of a cyclic stress-strain behavior via a phenomenological hardening law, in order to obtain an accurate stress prediction. In that study several kinematic hardening laws, of various complexity, were compared and evaluated from their ability to predict the springback of a simple U-bend example. The study revealed that the Yoshida-Uemori hardening model [6] gave the best results. Therefore, the Yoshida-Uemori kinematic hardening model is used in this work. For a detailed explanation of this model, see Refs. [1][2][6].

The Yoshida-Uemori model includes four unknown material parameters in our implementation. It should be emphasized that this is a slightly modified version compared to the original model [6]. These parameters are determined from inverse modeling of a three point bending test, where the Mean Squared Error (MSE) between the predicted and experimental force-displacement relationships is minimized. For a detailed description of the material parameter identification procedure, see Refs. [2][3]. In Ref. [1], the material parameter identification is performed in the rolling direction exclusively. The obtained material parameters and MSE-values are presented in Table 2 below. The number of bending/unbending cycles the sheet material can be anticipated to be subjected to during a forming operation was judged to be something between zero and two. We have here chosen to fit the results to two bending cycles. It should be noted that the resulting hardening parameters to a certain extent are depending on if e.g. one or two cycles are chosen.

One of the main purposes of the present study was to investigate whether the parameters identified in the rolling direction also can be used to describe the behavior in other directions. The other directions in this case are the transverse and diagonal directions.

When the principal response direction is different from the direction in which the material parameters have been determined, the planar anisotropy of the material will play an important role. Therefore the choice of yield function can be anticipated to be of considerable importance.

With this in mind, three-point bending tests were also performed in the transverse and diagonal directions, and the FE-results were fitted to the cyclic force-displacement curves. All the three different yield functions discussed

TABLE 2. Mean-squared error and material parameters for the Yoshida-Uemori hardening model. (two cycles).

\begin{tabular}{llllll}
\hline Material & $\boldsymbol{C}_{\boldsymbol{x}}$ & $\boldsymbol{b}$ & $\boldsymbol{k}$ & $\boldsymbol{h}$ & $\boldsymbol{M S E}$ \\
\hline TKS-DP600 & 295.1 & 167.0 & 90.13 & 0.361 & 0.00216 \\
TKS-220IF & 463.7 & 79.88 & 292.7 & 0.9267 & 0.07194 \\
\hline
\end{tabular}


TABLE 3. Material: TKS-DP600. MSE-values for the three different yield criteria (two cycles).

\begin{tabular}{lccc}
\hline Direction & Hill'48 & Barlat-Lian (Yld89) & Banabic/Aretz (BBC2005) \\
\hline Rolling (00) & 0.00215 & 0.00217 & 0.00216 \\
Transverse (90) & 0.00498 & 0.00514 & 0.00185 \\
Diagonal (45) & 0.00248 & 0.00254 & 0.00218 \\
\hline
\end{tabular}

TABLE 4. Material: TKS-220IF. MSE-values for the three different yield criteria (two cycles).

\begin{tabular}{lccc}
\hline Direction & Hill'48 & Barlat-Lian (Yld89) & Banabic/Aretz (BBC2005) \\
\hline Rolling (00) & 0.0785 & 0.0759 & 0.0719 \\
Transverse (90) & 0.0880 & 0.0736 & 0.0725 \\
Diagonal (45) & 0.0736 & 0.0837 & 0.0724 \\
\hline
\end{tabular}

above were applied. In all cases the Yoshida-Uemori hardening law with material parameters determined in the rolling direction were used. The resulting MSE-values for each material are presented in Table 3 and Table 4 below.

\section{HYSTERESIS EFFECTS IN AN UNLOADING/RELOADING CYCLE}

\section{Introductory Remarks}

The amount of springback during unloading depends also to a great extent on the elastic stiffness of the material. In classic elastoplasticity theory, the unloading of a material after plastic deformation is assumed to be linearly elastic with the stiffness equal to Young's modulus. However, several experimental investigations have revealed that this is an incorrect assumption.

The other main object of the present study has been to evaluate the unloading behavior at various levels of plastic strain for the considered materials. Below follows a short review of the observations from this evaluation.

\section{Experimental Observations}

The mechanical testing of the unloading behavior was performed with an ordinary tension test machine, with a video extensometer for measurement of the strains. A test specimen, $140 \times 20 \mathrm{~mm}$, was loaded to a certain plastic strain level and then unloaded. After the unloading phase the material was reloaded up to a new plastic strain level, and then the specimen was again unloaded. This procedure was repeated at seven different plastic strain levels: $1 \%$, $2 \%, 4 \%, 6 \%, 8 \%, 11 \%$, and $14 \%$. An illustration of a typical resulting stress-strain relationship is presented in Figure 1a.

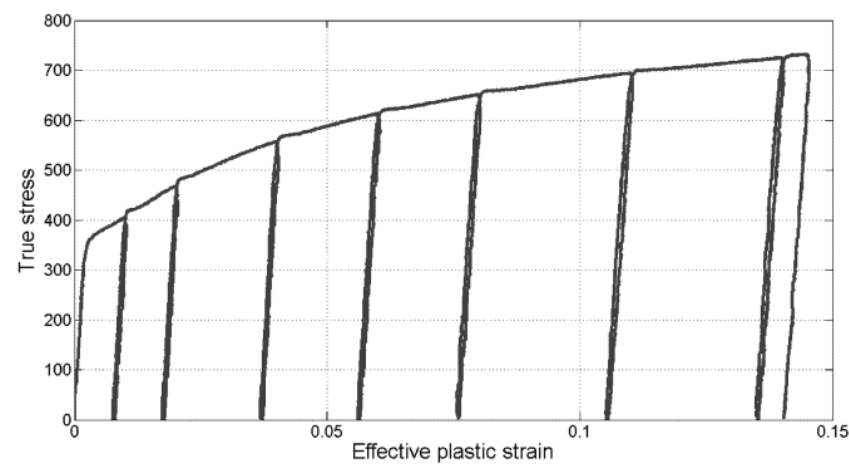

a.

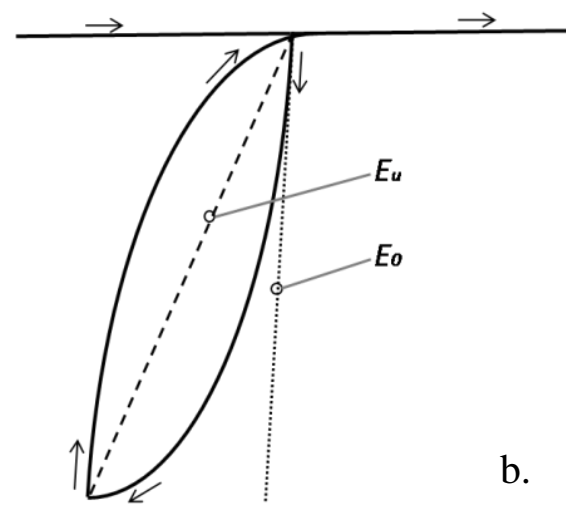

FIGURE 1. a: Experimental, uniaxial loading-unloading-reloading curve for the TKS-DP600 material, b: A detailed illustration of the unloading-reloading relationship. Material: TKS-DP600. 
A closer look at the unloading-reloading phase (Figure 1b) shows that there is a difference between the unloading and reloading curves. The material shows a non-linear unloading behavior. The curves deviate from linearity around an imaginary "mean" line, representing the secant to the curves. In classical elastoplastic theory, this secant is assumed to be equal to the initial elastic (Young's) modulus. However, the experiments evaluated in connection to this report, clearly show that the slope of this secant is strongly affected by the amount of plastic strain. More precisely, the magnitude of the unloading modulus is decreasing with increasing plastic strain.

At each plastic strain level demonstrated in Figure 1a, the slope of the secant to the unlading-reloading curves, $E_{u}$ in Figure 1, is measured. Hereafter, the slope $E_{u}$ will be denoted the "unloading modulus".

For the modeling of the unloading behavior of the materials, it is convenient to describe the experimental observations with a mathematical expression. As mentioned earlier, several different expressions have been proposed. However, the most common ones involve some kind of exponential expression. In this work an analytical expression proposed by Yoshida et al. [5] was used:

$$
E_{u}=E_{0}-\left(E_{0}-E_{s a t}\right) \cdot\left(1-e^{-\xi \cdot \bar{\varepsilon}^{p}}\right)
$$

where $E_{0}$ is the initial Young's modulus, $E_{\text {sat }}$ is a value that the unloading modulus saturates towards, and $\xi$ is a material parameter.

The values of the initial Young's modulus, $E_{0}$, were determined by a resonant frequency technique. The saturated values, $E_{\text {sat }}$, and the material parameters $\xi$ were determined by an optimization routine such that the Mean Squared Errors between the experimental values and the analytical expression, Eq. (6), were minimized.

In the current FE-analyses the unloading modulus was used in the material model instead of the initial Young's modulus, thus neglecting the fact that the unloading and reloading curves are nonlinear according to Figure 1.

\section{Results}

The unloading modulus functions, Eq. 6, were determined for all considered materials and in the rolling, diagonal and transverse directions. Figure 2 shows the resulting unloading modulus degradation with effective plastic strain in the rolling direction. It should be mentioned that there are very small differences between the unloading modulus degradation in the rolling-, diagonal and transverse directions respectively, for the considered materials.

\section{SIMULATION OF SPRINGBACK OF THE U-BEND PROBLEM}

The well known U-bend benchmark from the NUMISHEET'93 conference is used as an evaluating example of the springback. The experimental set-up is described in Figure 4 below. Just as for the simulation of the three-point bending test, the FE-code LS-DYNA was used to solve the problem. The forming step was solved by means of an explicit, dynamic solver, and the springback step with an implicit one. The symmetry of the problem was utilized, and only one quarter of the model was analyzed. The geometry of the tools is shown in Figure 4a, and the total size of the blank was $300 \times 50 \mathrm{~mm}$. The blankholder force was chosen to $40-100 \mathrm{kN}$, depending on the material. Fully

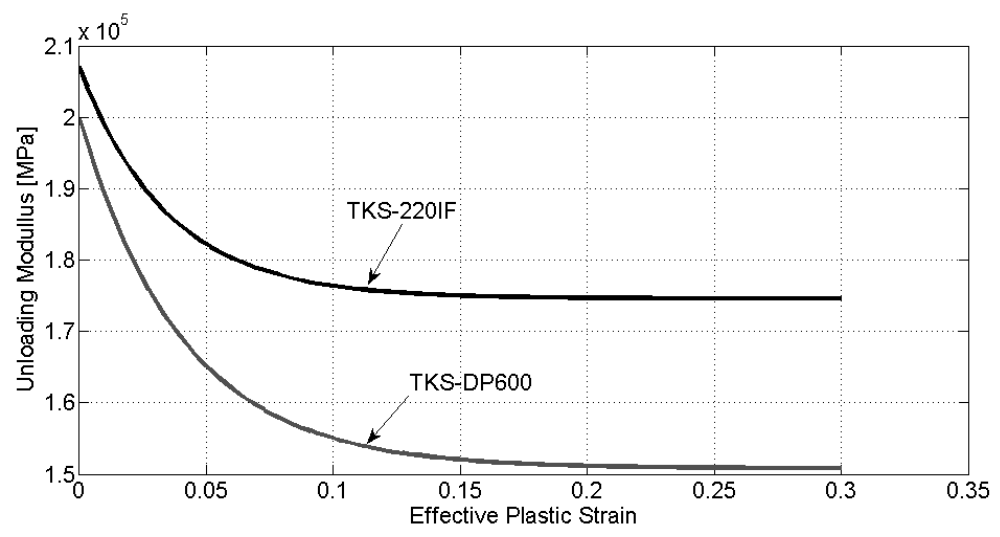

FIGURE 2. Unloading modulus degradation for the two materials in the rolling direction. 
integrated quadrilateral shell elements were utilized in the FE-model.

As shown in the work by Mattiasson et al. (1995) [11], the stress distribution in the workpiece after forming is strongly dependent on the element size in the finite element model of the sheet material. For a too coarse mesh, there is a pronounced stress relaxation after the material has reached its peak stress point at the exit of the die radius. Therefore, a comprehensive mesh convergence study was performed. Convergent results were obtained with a mesh size of $1 \times 2 \mathrm{~mm}$ and with nine integration points through the sheet thickness.

Just as for the three-point bending test, the specimens for the springback experiment were cut out from the coil in three different directions: The rolling-, transverse- and diagonal directions respectively. All experiments were made three times in order to eliminate influence of scatter.

\section{Influence of the Unloading Modulus and Kinematic Hardening Law}

As the authors recently have pointed out, [1][2], the unloading modulus and the kinematic hardening law have a significant effect on the quality of the springback prediction. Figure 4 shows the effect of using the unloading modulus, according to Eq. 6, and the effect of kinematic hardening law on the final springback prediction for the TKS-DP600 material. In all these analyses the 8-parameter yield criterion by Banabic/Aretz has been used. It is obvious that both these effects play a significant role. The Yoshida-Uemori and the Geng-Wagoner models deviate less than two percents from the experimental data when the unloading modulus is used. For the three other hardening models, the deviation is more significant. Without taking the unloading modulus into consideration, the springback prediction is under-predicted for all kinematic hardening laws, and the best result is obtained with the simple isotropic hardening law.

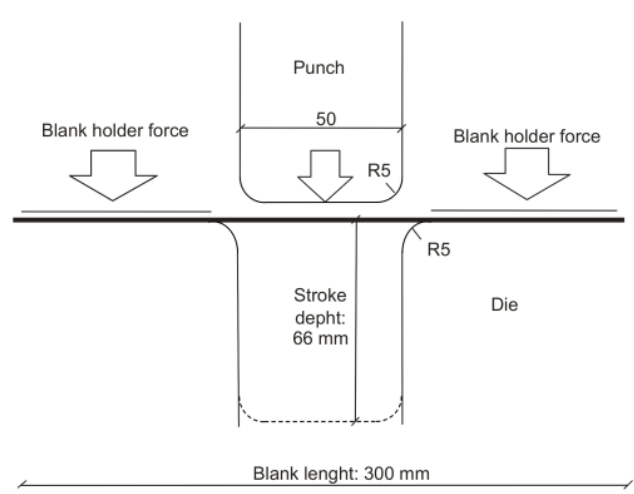

(a)

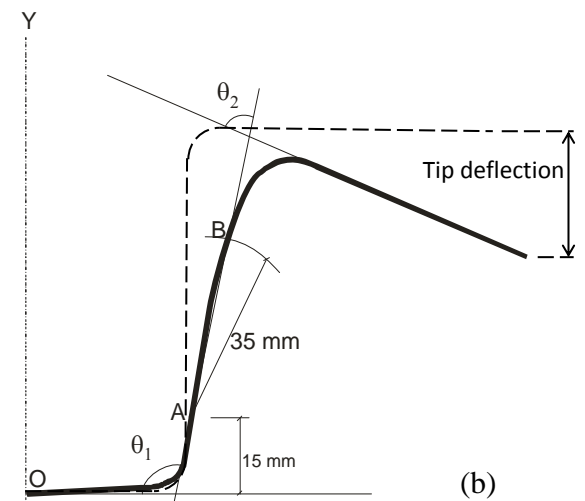

FIGURE 3. The NUMISHEET'93 benchmark problem. (a) Experimental set-up; (b) Definition of the tip deflection, used for the evaluation of the springback.

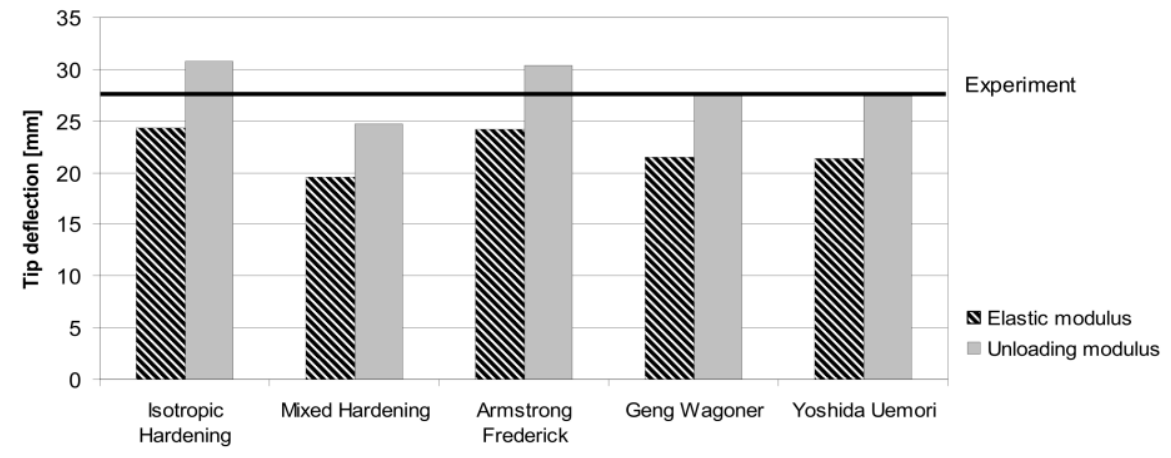

FIGURE 4. Material TKS-DP600. Predicted tip deflection after springback with and without consideration of elastic stiffness degradation, for the 8-parameter yield criterion by Banabic/Aretz. 


\section{Influence of the Yield Criterion and the Orientation of the Sheet}

The springback behavior was evaluated in the rolling-, transverse- and diagonal directions for all considered materials, and for all the three different yield criteria. Since the Yoshida-Uemori hardening law has been proven to describe the cyclic behavior best (see. Figure 4 and Refs. [1][2]), this hardening law is used in all predictions presented in this section. The hardening parameters in the Yoshida-Uemori hardening law are obtained from the three-point bending test when the sheet is aligned in the rolling direction (see Table 2). Furthermore, since consideration of the unloading modulus is necessary for accurate springback prediction (Figure 4, Refs. [1][2]) the unloading modulus according to Eq. 6 is used for all predictions. Figure 5 shows some of the results from this study. The figure illustrates the tip deflections in staple diagrams for the two materials described in Table 1. The transparent rectangular areas indicate experimental values including scatter.

As can be seen from the results, the scatter in the experimental springback is between $2-5 \%$ depending on the material.

All the yield criteria give fairly good predictions in at least one direction. However, it is only the Banabic/Aretz (BBC2005) criterion that seems to give accurate springback predictions in all three considered directions. The predictions for this criterion are inside or very close to the experimental interval for all materials and all directions. This is also what can be expected based on the MSE-values from the three-point bending test (see Table 3 and Table 4).

\section{Worst Case Scenario}

As can be seen from the results presented earlier in this chapter, the quality of the springback predictions is highly dependent on the choice of yield criteria and kinematic hardening law. Based on these results one can think of combinations that constitutes "worst case scenarios". As an example, for the TKS-DP600 material, the Hill'48 yield criterion over-predicts the springback magnitude in the transverse direction. If this is combined with pure isotropic hardening that also over-predict the springback, an upper limit of the springback is obtained. In a similar fashion, for the same material and direction, one can chose the Banabic/Aretz yield criterion in combination with the mixed hardening law. In this case a lower limit of the springback prediction is obtained. All other combinations will then yield results somewhere in between these upper and lower limits. An illustration of this is presented in Figure 6. The grey area represents the interval between the upper and lower limits of the springback geometries. In between these limits the springback for Banabic/Aretz yield criterion together with Yoshida-Uemori kinematic hardening is illustrated together with experimental data.
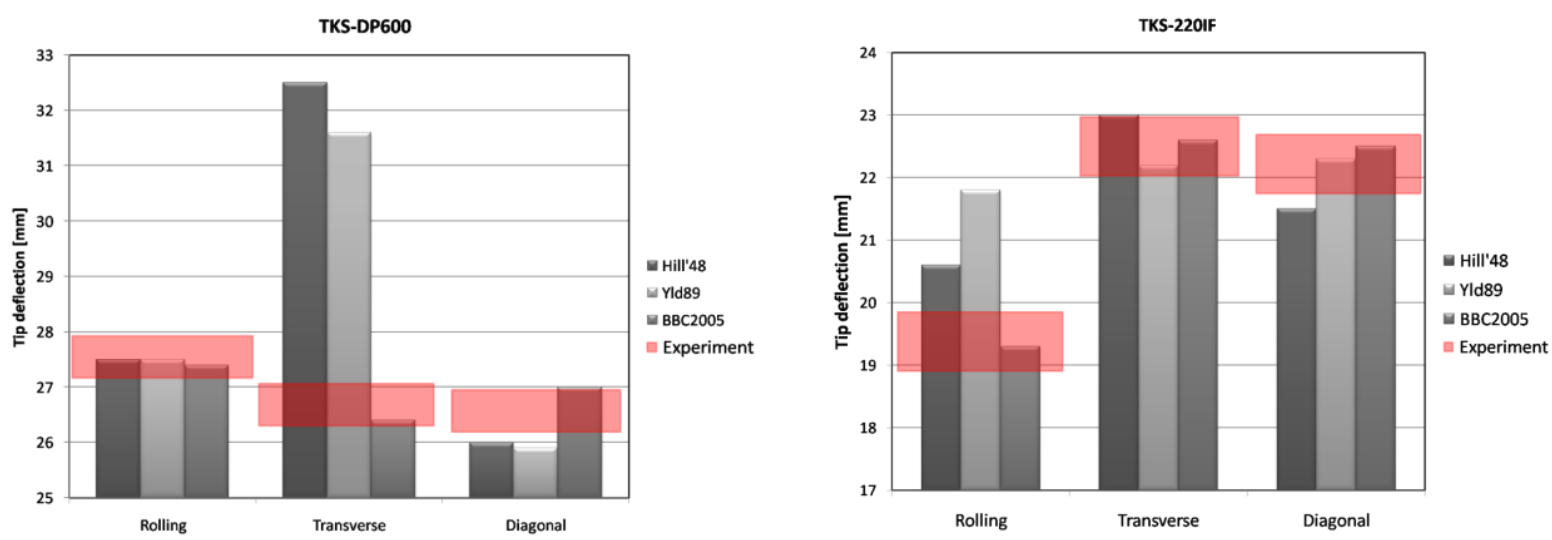

FIGURE 5. Effect of yield criterion. Springback deflection of the tip in three directions. Material: Left: TKS-DP600, Right: TKS-220IF. 


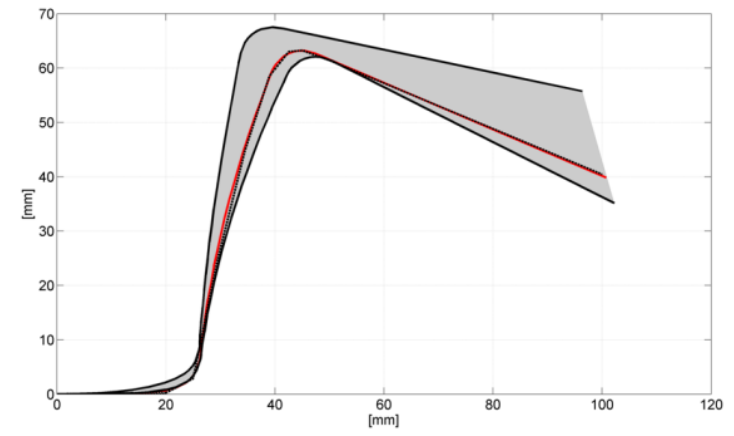

FIGURE 6. Illustration of a possible interval for predicted springback. Material: TKS-DP600 in the transverse direction.

\section{CONCLUDING REMARKS}

The authors have recently, Ref. [1], showed the importance of the choice of kinematic hardening law and the importance of taking the unloading modulus in account, in order to get accurate springback predictions. In this article, also the importance of the choice of yield criterion is demonstrated. Three different yield criteria were used to express the anisotropic behavior of the materials: The Hill'48 yield criterion, the Barlat-Lian (Yld89) criterion and the 8-parameter yield criterion by Banabic/Aretz (BBC2005). Springback and three-point bending tests were performed in the rolling-, transverse- and diagonal directions for four materials commonly used in the automotive industry.

From the current study the following important conclusions can be drawn:

- Material hardening parameters, determined from bending tests in one specific direction, can with acceptable accuracy be used for loadings in other directions.

- The results clearly show that for accurate springback prediction, the BBC2005 yield criterion is the only one of the three tested criteria that can give acceptable springback results in all directions. The other two criteria work in general quite well, as long as the loading direction coincides with the rolling direction. However, if the sheet is aligned in another direction, acceptable results cannot be guaranteed with the two simpler models

- The degradation of elastic stiffness due to plastic straining is probably the single constitutive ingredient that has the greatest impact on the predicted springback. For the four tested materials the saturated unloading modules were 14-28\% lower than the corresponding Young modules.

- When the "best" (most advanced) yield condition and hardening law has been used in combination, excellent predictions of the springback has been achieved for all the materials and in all directions.

- It has also been demonstrated that improper choices of yield criteria and kinematic hardening laws can result in a wide spread in the predicted springback results. However, inappropriate choices of constitutive ingredients can, in some instances, still result in accurate springback predictions in cases when the effects of several errors cancel out each other.

\section{ACKNOWLEDGMENTS}

The characterization of the materials used in this study was conducted by Per Thilderkvist and Jörgen Hertzman at the Industrial Development Center in Olofström, Sweden. The three-point bending tests were performed by Bertil Enquist at Växjö University. Their contribution to this work is gratefully acknowledged.

The work has been performed within the Swedish national research program MERA (Manufacturing Engineering Research Area). Financial support has been provided by Vinnova.

\section{REFERENCES}

1. P.A. Eggertsen and K. Mattiasson. Int. J. of Mech. Sci. 51, 2009, pp. 547-563.

2. P.A. Eggertsen. Licentiate thesis, Chalmers University of Technology (2009).

3. P.A. Eggertsen and K. Mattiasson. Eng. With Computer. DOI 10.1007/s00366-009-0149-y. (2009)

4. K. Mattiasson and M. Sigvant. Int. J of Mech. Sci. 50, 2008, pp. 774-787. 
5. F. Yoshida, T. Uemori and K. Fujiwara. Int. J of Plasticity 18, 2002, pp. 633-659.

6. F. Yoshida and T. Uemori. Int. J of Mech. Sci. 45, 2003, pp. 1687-1702.

7. R. Hill. Proc. of the Royal Soc.A193, 1948, pp. 145-85.

8. F. Barlat and J. Lian. Int. J of Plasticity 5, 1989, pp. 51-66.

9. R.W. Logan and W.F. Hosford. Int. J. of Mech. Sci. 27, 1980, pp. 419-430.

10. D. Banabic and H. Aretz. Int. J of Plasticity 21, 2005, pp. 493-512.

11. K. Mattiasson, A. Strange, P. Thilderkvist, A. Samuelsson. NUMIFORM'95, Sim. Of Mat. Proc: Theory, Methods and Applications. 1995, pp. 115-124.

12. F. Barlat, J.C. Brem, J.W. Yoon, K. Chung, et al. Int. J of Plasticity 19, 2003, pp. 1297-1319.

13. H. Aretz. J. of Mat. Proc. Tech. 168, 2005, pp. 1-9. 